\section{S. Webendörfer \\ P. Messerer \\ F. Eberle \\ A. Zober}

\title{
Darmkrebs-Vorsorge im Betrieb
}

\author{
Eine Initiative sekundärer Prävention in der \\ BASF Aktiengesellschaft ${ }^{1}$
}

\section{Screening for colorectal cancer: an initiative for secondary prevention at the BASF Aktiengesellschaft}

\begin{abstract}
Hintergrund und Fragestellung: In Deutschland wird die Zahl der jährlichen Neuerkrankungen an Darmkrebs auf etwa 27000 bei Männern und 30000 bei Frauen geschätzt. Bei frühzeitiger Diagnosestellung liegt die Heilungschance bei über $90 \%$. Vor diesem Hintergrund hat die Abteilung Arbeitsmedizin und Gesundheitsschutz der BASF Aktiengesellschaft am Standort Ludwigshafen im Sinne ihres Präventionsauftrages eine Vorsorgeinitiative gegen Darmkrebs durchgeführt.
\end{abstract}

Probanden und Methodik: Zielgruppe waren alle 13265 aktiv beschäftigten Mitarbeiter ab dem 45. Lebensjahr. Interessierte erhielten einen standardisierten Fragebogen zu Risikofaktoren für Darmkrebserkrankungen und den Fecal-Occult-Blood-Test ( FOBT). Bei positivem Testergebnis und/oder der Beantwortung einer der Fragen nach Blut im Stuhl bzw. positiver Familienanamnese mit „JA“ wurde nach den Empfehlungen der Deutschen Gesellschaft für Verdauungs- und Stoffwechselerkrankungen, DGVS, eine endoskopische Abklärung mittels Koloskopie über den Hausarzt angeraten.

Ergebnisse: Bei Abschluss der Aktion lagen von 3732 Mitarbeitern (337 Frauen, 3395 Männer, mittleres Alter 52 Jahre) Testergebnisse und ein vollständig ausgefüllter Fragebogen vor. 688 Mitarbeitern wurde eine Koloskopie empfohlen - 323 Teilnehmer (47\%) ließen diese Untersuchung durchführen. Bei neun Mitarbeitern lagen bereits manifeste Karzinome vor, sechs davon wurden noch in den Frühstadien T1 oder T2 entdeckt. In 61 Fällen wurden adenomatöse Polypen diagnostiziert und anschließend abgetragen. Kosten-Nutzenbetrachtungen auf betrieblicher Ebene bzw. im Bereich der Gesundheitssysteme ergaben günstige Relationen von 1:10 bzw. 1:14.

Folgerung: Nur mit großem Aufwand ist es möglich, die Teilnahmerate an Krebsvorsorgeuntersuchungen zu erhöhen und die konsequente Abklärung auffälliger Befunde zu erreichen. Dabei ist die betriebliche Gesundheitsvorsorge eine wertvolle Ergänzung zur haus- und fachärztlichen Versorgung in Deutschland, wenn eine enge Zusammenarbeit mit den niedergelassenen Fachkollegen in der Region gewährleistet ist. Aktionen wie diese bewahren die individuell Betroffenen vor persönlichem Leid und sind auch wirtschaftlich ein Erfolg.
Background and objective: The annual incidence of colorectal cancer in Germany is estimated at 27000 in men and 30000 in women. If the diagnosis is made early the cure rate is over $90 \%$. Against this background the department of occupational medicine and health protection of the BASF Aktiengesellschaft initiated a study on the potential prevention of colorectal cancer among the staff at its Ludwigshafen site.

Subjects and methods: The target group included all 13265 actively working employees aged 45 years or above. Those expressing interest were given a standardized questionnaire concerning risk factors for colorectal cancer and a test for occult fecal blood (FOBT). If the test was positive and/or a positive answer was given to the question on blood in the stool or on a positive family history, coloscopy - to be arranged via the general practitioner - was advised, in line with the recommendations of the German Society of Digestive and Metabolic diseases (Deutsche Gesellschaft für Verdauungs- und Stoffwechselerkrankungen).

Results: At the end of the study 3732 employees (337 women, 3395 men, mean age 52 years) had completed the questionnaire and the FOBT results were available. Colposcopy was recommended to 688 employees, 323 of whom (47\%) underwent the investigation. Nine of the subjects already had manifest cancer, six of them in the early stage $\mathrm{T} 1$ or $\mathrm{T} 2$. Adenomatous polyps were found in an additional 61 and subsequently excised. Costbenefit considerations at the company level or in the area of the health system, respectively, gave favourable ratios of 1:10 and 1:14. Conclusion: It requires considerable expenditure to increase the number of participants in the cancer prevention programme and obtain a consequent clarification of suspicious findings. Health care within a company is a valuable complementation in Germany of medical care provided by general practitioners or specialist, if close cooperation between practitioners in the given region is assured. Initiatives like the one presented here protect people personally affected against pain and distress and are also of economic value.

1 Diese Initiative wurde in Kooperation mit der Stiftung LebensBlicke - Früherkennung Darmkrebs, Ludwigshafen/Rhein durchgeführt BASF-Aktiengesellschaft, Ludwigshafen/Rhein 
In Deutschland wird die Zahl der Neuerkrankungen an Darmkrebs pro Jahr auf etwa 27000 bei Männern und 30000 bei Frauen geschätzt. Darmkrebs ist damit bei Männern die dritthäufigste, bei Frauen die zweithäufigste Krebserkrankung und sowohl für Männer als auch für Frauen nach Lungen- bzw. Brustkrebs die zweithäufigste Krebstodesursache $(1-5,7,12,13)$. Die Inzidenz wird bei Männern im Alter von 45-59 Jahren auf 56,6 pro 100000 Personen geschätzt $(1,3,7,13)$. Die 5-Jahres-Überlebensrate liegt bei etwa $50 \%(2,13)$ und kann bei frühzeitiger Diagnosestellung und z. B. endoskopischer Therapie auf über $90 \%$ gesteigert werden (5).

Präventivmedizin ist ein wesentlicher Inhalt der Arbeitsmedizin. Vor diesem Hintergrund nutzen die Werksärzte der BASF die Nähe zu den Mitarbeitern des Unternehmens, um kontinuierlich sowohl über alle Möglichkeiten der Früherkennung und Heilung bösartiger Erkrankungen aufzuklären als auch entsprechende Initiativen zu ergreifen. Der Darmkrebs ist mit seinen hohen Heilungschancen bei rechtzeitiger Erkennung ein besonders gutes Beispiel für eine erfolgversprechende sekundäre Prävention. Die Vorsorgeinitiative verfolgte zum einen das Ziel, den Mitarbeitern der BASF in Ludwigshafen ein zusätzliches Angebot zur Gesundheitsvorsorge zu machen und zum anderen, durch die begleitende Öffentlichkeitsarbeit die Belegschaft generell für das Thema Früherkennung von Darmkrebs zu sensibilisieren.

\section{Probanden und Methodik}

Zielgruppe waren alle 13265 Beschäftigte (1618 Frauen, 11647 Männer) am Standort Ludwigshafen im Alter von 45 Jahren oder älter. Auf die Aktion und das Thema Darmkrebsfrüherkennung wurde durch die Werkszeitung, das firmeneigene Intranet, Plakate, persönliche Anschreiben und im Gespräch mit den BASF-Werksärzten aufmerksam gemacht. Alle Interessierten erhielten im Zeitraum vom 1. November 2001 bis 31. Juli 2002 Informationsschreiben zum Thema Darmkrebs sowie über den „Fecal-Occult-Blood“Test (FOBT) - Haemoccult ${ }^{\circledR}$. Ein Anamnese-Fragebogen und die Testbriefchen wurden den interessierten Mitarbeitern persönlich ausgehändigt oder per Werkpost zugeschickt.

Im ersten Teil des Fragebogens wurde nach sichtbaren Blutauflagerungen im Stuhl und der familiären Belastung mit Darmkrebs bei einem Verwandten ersten Grades, im zweiten Teil nach einer Veränderung des Körpergewichtes, veränderten Stuhlgewohnheiten und allgemeinen Verdauungsbeschwerden gefragt. Der dritte Teil ging auf die Eigenanamnese im Hinblick auf vorbekannte chronisch entzündliche Darmerkrankungen, zurückliegende FOBT-Ergebnisse, bereits erfolgte endoskopische Darmuntersuchungen und die aktuelle Medikamentenanamnese ein.

Die zurückgegebenen Testbriefchen wurden im klinisch-chemischen Labor der Abteilung Arbeitsmedizin und Gesundheitsschutz ausgewertet. Erfolgte innerhalb von 14 Tagen keine Rückgabe der Testbriefchen ans Labor, wurden die entsprechenden Teilnehmer mindestens einmal per Telefon oder E-mail an die Abgabe erinnert. Die Testergebnisse erhielten alle Teilnehmer in einem persönlichen Anschreiben.

Bei positivem FOBT und/oder bei Beantwortung einer der Fragen nach Blut im Stuhl bzw. bezüglich einer positiven Familienanamnese mit "JA“ wurde den Betroffenen schriftlich nach
Tab.1 Charakteristika der Kollektive.

\begin{tabular}{|llll}
\hline Gruppe & $\begin{array}{l}\text { Zielgruppe } \\
\text { (n= 13265) }\end{array}$ & $\begin{array}{l}\text { Interessenten } \\
\text { (n=4172) }\end{array}$ & $\begin{array}{l}\text { Teilnehmer } \\
\text { (n=3732) }\end{array}$ \\
\hline $\begin{array}{l}\text { Deutsche } \\
\text { Nicht-Deutsche }\end{array}$ & $862(6,5 \%)$ & $284(6,8 \%)$ & $265(7,1 \%)$ \\
\hline $\begin{array}{l}\text { Frauen } \\
\text { Männer }\end{array}$ & $1618(12,2 \%)$ & $388(9,3 \%)$ & $337(9,0 \%)$ \\
$\begin{array}{l}\text { Gewerbliche Mitarbeiter } \\
\text { darunter in vollkontinu- } \\
\text { ierlicher Wechselschicht }\end{array}$ & $5055(38,1 \%)$ & $1520(36,4 \%)$ & $1345(36,0 \%)$ \\
\hline $\begin{array}{l}\text { Tarifliche Mitarbeiter } \\
\text { darunter in vollkontinu- } \\
\text { ierlicher Wechselschicht }\end{array}$ & $5631(420,5 \%)$ & $964(23,1 \%)$ & $851(22,8 \%)$ \\
$\begin{array}{l}\text { Außertarifliche } \\
\text { Mitarbeiter }\end{array}$ & $2579(19,4 \%)$ & $1700(40,8 \%)$ & $1520(40,7 \%)$ \\
\hline
\end{tabular}

den Empfehlungen der Deutschen Gesellschaft für Verdauungsund Stoffwechselerkrankungen, DGVS, (12) eine endoskopische Abklärung mittels Koloskopie über den Hausarzt angeraten. Die Betroffenen wurden gebeten, dem Haus- oder Facharzt ihr Einverständnis zu geben, die Untersuchungsbefunde an die Abteilung Arbeitsmedizin und Gesundheitsschutz weiterzuleiten, um eine Auswertung der Ergebnisse zu ermöglichen.

Bei Beantwortung einer der Fragen nach Veränderung des Körpergewichtes , geänderten Stuhlgewohnheiten und allgemeinen Verdauungsbeschwerden mit "JA“ wurde den Betroffenen lediglich die Vorstellung beim Hausarzt empfohlen. Dieser stellte eigenverantwortlich unter Berücksichtigung der aktuellen Untersuchungs- und Vorbefunde die Indikation zu weiteren Untersuchungen. Der negative FOBT und die unauffällige Anamnese stellten den Normalbefund dar. In diesen Fällen wurde die Wiederholung der Krebsvorsorgeuntersuchung nach Ablauf von 12 Monaten empfohlen.

Es wurde regelmäßig an noch ausstehende Untersuchungsergebnisse erinnert, sodass am Ende der Aktion für jeden abklärungsbedürftigen Befund eine Rückmeldung vorlag. Entweder hatte der weiterbehandelnde Arzt das Koloskopie-Ergebnis übersandt, oder der Teilnehmer hatte mitgeteilt, aus welchem Grund die endoskopische Diagnostik nicht erfolgt war.

\section{Ergebnisse}

Die Anzahl der Interessenten an der Aktion betrug insgesamt 4172 (388 Frauen, 3784 Männer). Das entspricht 24\% bzw. 32,5\% der jeweiligen Zielgruppe an aktiven Mitarbeiterinnen bzw. Mitarbeitern der BASF-Aktiengesellschaft am Standort Ludwigshafen ab dem 45. Lebensjahr. Tab.1 zeigt die Verteilung nach Nationalität, Geschlecht und Personalverwaltungsschlüssel.

Trotz Erinnerung schickten 10,5\% derjenigen, die ursprünglich Interesse an der Aktion bekundet hatten, weder einen ausgefüllten Fragebogen noch die Testbriefchen zurück.

Das mittlere Alter (Median) der Teilnehmer betrug 52 Jahre. Bei Männern und Frauen, den Personen in den verschiedenen Beschäftigungsverhältnissen oder Arbeitszeitformen bestanden keine bedeutsamen Unterschiede in der Verteilung zwischen Zielgruppe und Teilnehmern. Lediglich Frauen schienen gegenüber ihrem Anteil in 
Tab.2 Altersverteilung der Teilnehmer mit positivem FOBT.

\begin{tabular}{|c|c|c|c|c|c|c|}
\hline \multirow{2}{*}{$\begin{array}{l}\text { Altersgruppe } \\
\text { (Jahre) }\end{array}$} & \multicolumn{3}{|l|}{ Männer } & \multicolumn{3}{|l|}{ Frauen } \\
\hline & $n=3395$ & $\begin{array}{l}\text { FOBT+ } \\
n=264\end{array}$ & $\mathrm{FOBT}+(\%)$ & $\mathrm{n}=337$ & $\begin{array}{l}\text { FOBT+ } \\
n=19\end{array}$ & $\mathrm{FOBT}+(\%)$ \\
\hline $45-49$ & 1230 & 66 & 5,4 & 118 & 6 & 5,1 \\
\hline $50-54$ & 1143 & 93 & 8,1 & 122 & 7 & 5,7 \\
\hline $55-59$ & 862 & 91 & 10,6 & 82 & 4 & 4,9 \\
\hline$>60$ & 160 & 14 & 8,7 & 15 & 2 & 13,3 \\
\hline
\end{tabular}

der Zielgruppe etwas unterrepräsentiert. Das ist möglicherweise dadurch bedingt, dass Krebsvorsorgeuntersuchungen vermehrt im Rahmen gynäkologischer Kontrollen beim Facharzt erfolgen.

\section{„Fecal-Occult-Blood“-Test}

Der FOBT war bei 264 (7,8\%) der 3395 teilnehmenden Männer und bei $19(5,6 \%)$ der 337 teilnehmenden Frauen positiv. In Tab.2 ist die Altersverteilung bei positivem FOBT dargestellt.

Sowohl bei Männern als auch bei Frauen war der Anteil der positiven Ergebnisse im FOBT bezogen auf die Teilnehmerzahl einer Altersgruppe erwartungsgemäß bei den 45 - bis 49-Jährigen am niedrigsten. Für beide Geschlechter stieg er in der Altersgruppe von 50-54 Jahren auf $8,1 \%$ bzw. 5,7\% an. In der Folge zeigte sich ein weiterer Anstieg nur bei den Männern bis einschließlich 59 Jahre.

\section{Koloskopie}

Die Empfehlung zur Abklärung mittels Koloskopie erfolgte ausschließlich nach den Leitlinien der Deutschen Gesellschaft für Verdauungs- und Stoffwechselerkrankungen (DGVS) (12) bei 688 Teilnehmern, darunter 621 Männer und 67 Frauen.

Tab.3 zeigt die Anzahl der Teilnehmer, die sich einer Koloskopie unterzogen haben sowie die Verteilung nach den Gründen, die zur Koloskopieempfehlung geführt haben.

Insgesamt stellten sich 142 von den 405 Teilnehmern (35,1\%), die eine positive Familienanamnese und/oder Blut im Stuhl bemerkt hatten (Frage 1/2 im Fragebogen), beim Haus-, oder Facharzt zur Koloskopie vor. 146 der 229 Teilnehmer (63,8\%), die ausschließlich einen positiven FOBT aufwiesen, ließen sich koloskopieren. Bei 35 von 54 Teilnehmern (64,8\%), die sowohl einen positiven FOBT und eine familiäre Belastung bzw. Blut im Stuhl bemerkt hatten, erfolgte eine Koloskopie. 365 Teilnehmer (53,1\%), 329 Männer (53,0\%) und 36 (53,7\%) Frauen, ließen die Untersuchung nicht durchführen.

In Tab.4 sind die von den Teilnehmern genannten Begründungen für nicht durchgeführte Koloskopien dem Anlass zur Koloskopieempfehlung gegenüber gestellt. In 77 Fällen wurde die Untersuchung nach Angaben der Teilnehmer vom Hausarzt trotz schriftlicher Empfehlung im Rahmen der Vorsorgeaktion nicht veranlasst - 288 Teilnehmer lehnten die Diagnostik von sich aus ab. In 33 Fällen wurde der FOBT vom Hausarzt kontrolliert. Nach dem negativen Ausfall des FOBT erfolgte keine weitergehende Diagnostik. In sechs Fällen wurde die Diagnose „Hämorrhoiden“, in elf Fällen das alleinige Vorliegen der positiven Familienanamnese als Begründung für die Nichtveranlassung der Koloskopie angeführt.
Tab.3 Teilnehmer mit Koloskopie und Häufigkeit der Gründe zur Koloskopieempfehlung.

\begin{tabular}{|c|c|c|c|c|}
\hline \multirow{2}{*}{$\begin{array}{l}\text { Gründe für die } \\
\text { Koloskopie- } \\
\text { empfehlung }\end{array}$} & \multicolumn{2}{|c|}{ Koloskopie-Empfehlung } & \multicolumn{2}{|c|}{ Koloskopie durchgeführt } \\
\hline & $\begin{array}{l}\text { Männer } \\
n=621\end{array}$ & $\begin{array}{l}\text { Frauen } \\
n=67\end{array}$ & $\begin{array}{l}\text { Männer } \\
n=292\end{array}$ & $\begin{array}{l}\text { Frauen } \\
n=31\end{array}$ \\
\hline $\begin{array}{l}\text { Nur Frage } 1 \text { oder } 2 \\
\text { "Ja“ }\end{array}$ & 355 & 50 & $124(34,9 \%)$ & $18(36,0 \%)$ \\
\hline Nur FOBT positiv & 215 & 14 & $134(62,3 \%)$ & $12(85,7 \%)$ \\
\hline $\begin{array}{l}\text { FOBT positiv und } \\
\text { Frage } 1 \text { oder } 2 \text { "Ja“ }\end{array}$ & 51 & 3 & $34(62,9 \%)$ & $1(33,3 \%)$ \\
\hline \multicolumn{5}{|c|}{$\begin{array}{l}\text { FOBT: Fecal-Occult-Blood-Test } \\
\text { Frage 1: Haben Sie in den letzten } 6 \text { Monaten Blut im Stuhl bemerkt? } \\
\text { Frage 2: Gibt es in Ihrer Familie Dickdarmkrebs (Verwandte ersten Grades)? }\end{array}$} \\
\hline
\end{tabular}

Tab. 4 Gegenüberstellung von Begründungen für nicht durchgeführte Koloskopien und dem Anlass zur Koloskopieempfehlung.

\begin{tabular}{|c|c|c|c|c|}
\hline \multirow{2}{*}{$\begin{array}{l}\text { Begründung für nicht } \\
\text { durchgeführte } \\
\text { Koloskopien }(n=365)\end{array}$} & \multirow[t]{2}{*}{$n$} & \multicolumn{3}{|c|}{$\begin{array}{l}\text { Anlass zur Koloskopie- } \\
\text { empfehlung }\end{array}$} \\
\hline & & $\begin{array}{l}\text { FOBT+ } \\
\text { und } \\
\text { F1/2 }\end{array}$ & $\begin{array}{l}\text { Nur } \\
\text { FOBT+ }\end{array}$ & $\begin{array}{l}\text { Nur } \\
\text { F1/2 }\end{array}$ \\
\hline Arzt & $77(21,1 \%)$ & 4 & 39 & 34 \\
\hline FOBT kontrolliert: negativ & 33 & 3 & 26 & 4 \\
\hline $\begin{array}{l}\text { Koloskopie in Anamnese und } \\
\text { FOBT mit negativem Ergebnis } \\
\text { kontrolliert }\end{array}$ & 5 & 0 & 5 & 0 \\
\hline Marcumar-Behandlung & 2 & 0 & 2 & 0 \\
\hline Hämorrrhoiden & 6 & 0 & 1 & 5 \\
\hline Koloskopie in Anamnese o. B. & 11 & 1 & 2 & 8 \\
\hline Nur Familienanamnese positiv & 11 & 0 & 0 & 11 \\
\hline Keine Angabe eines Grundes & 9 & 0 & 3 & 6 \\
\hline Teilnehmer & $288(78,9 \%)$ & 15 & 44 & 229 \\
\hline Hämorrhoiden & 35 & 2 & 5 & 28 \\
\hline Koloskopie in Anamnese o. B. & 38 & 1 & 3 & 34 \\
\hline Unentschlossen & 56 & 3 & 7 & 46 \\
\hline Keine Angabe eines Grundes & 159 & 9 & 29 & 121 \\
\hline \multicolumn{5}{|c|}{$\begin{array}{l}\text { FOBT: Fecal-Occult-Blood-Test } \\
\text { Frage 1: Haben Sie in den letzten } 6 \text { Monaten Blut im Stuhl bemerkt? } \\
\text { Frage 2: Gibt es in Ihrer Familie Dickdarmkrebs (Verwandte ersten Gr }\end{array}$} \\
\hline
\end{tabular}

In Tab.5 wird die Anzahl der Koloskopiebefunde nach Diagnosen dargestellt.

In 70 Fällen fanden sich obligate Krebsvorstufen bzw. manifeste Krebserkrankungen. In 61 Fällen wurden in der endoskopischen Diagnostik adenomatöse Polypen festgestellt und entfernt. Damit konnte die Entwicklung einer karzinomatösen Entartung im Sinne der Adenom-Karzinom-Sequenz verhindert werden $(3,5,7,8,10)$. In der Altersgruppe unter 50 Jahren wurden 12 der 61 adenomatösen Polypen (19,7\%) entdeckt.

Bei neun Teilnehmern wurde die Diagnose eines Kolon- bzw. eines Rektumkarzinoms gestellt. Vier der Erkrankungen wurden im Stadium T 1, zwei im Stadium T 2 und drei im Stadium T 3 diagnostiziert. Ein Patient war 46 Jahre alt, die weiteren acht Pati- 
Tab.5 Koloskopiebefunde.

\begin{tabular}{|llll|}
\hline Koloskopiebefund & Gesamt $(\boldsymbol{n}=\mathbf{3 2 3})$ & $\begin{array}{l}\text { Männer } \\
(\mathbf{n = 2 9 2 )}\end{array}$ & $\begin{array}{l}\text { Frauen } \\
(\mathbf{n = 3 1 )}\end{array}$ \\
\hline Karzinom & 9 & 9 & 0 \\
\hline Polyp, adenomatös & 61 & 59 & 2 \\
\hline Polyp, hyperplastisch & 30 & 29 & 1 \\
Hämorrhoiden & 52 & 46 & 6 \\
\hline Divertikulose & 26 & 24 & 2 \\
\hline unauffällig & 145 & 125 & 20 \\
\hline
\end{tabular}

enten zwischen 50 und 59 Jahre alt (Median 52 Jahre). Bei sieben dieser Mitarbeiter war das Ergebnis des FOBT positiv gewesen. Drei Teilnehmer hatten zusätzlich sichtbare Blutauflagerungen im Stuhl bemerkt und ein Teilnehmer hatte zusätzlich eine positive Familienanamnese. In jeweils einem Fall führte allein die positive Familienanamnese bzw. allein die Beobachtung sichtbarer Blutauflagerungen im Stuhl zur Abklärung mittels Koloskopie und damit zur Diagnosestellung.

\section{Kosten-Nutzen-Relation}

Die Kosten-Nutzen-Relation wird getrennt für das Unternehmen (betriebswirtschaftliche Rechnung) und für das Gesundheitssystem (volkswirtschaftliche Rechnung) dargestellt: Nach Angaben des Bundesverbandes der Betriebskrankenkassen aus dem Jahr 1999 verursacht eine manifeste Darmkrebserkrankung eines Mitarbeiters im Mittel krankheitsbedingte Fehlzeiten von 60 Arbeitstagen. Damit ergibt sich bei 61 rechtzeitig entfernten Krebsvorstufen und durchschnittlichen Personalkosten pro Ausfalltag von EUR 313,- (nach Angaben des Personalwesens der BASF) eine Summe von ca. 1,1 Millionen EUR , die mittel- bis langfristig an Lohnfortzahlung aufgebracht werden müsste (für die ersten 30 Arbeitstage allein vom Arbeitgeber). Zusätzlich werden die Kosten für die sechs im Frühstadium diagnostizierten Karzinome gegenüber einer fortgeschrittenen Erkrankung geringer ausfallen. Die betriebsinternen Kosten für die Vorsorgeaktion in der BASF werden mit ca. 110 000,- EUR veranschlagt davon entfallen 95\% auf Personalkosten und 5\% auf Materialkosten. Damit beträgt die Kosten-Nutzen-Relation 1:10 bzw. wenn man den vom Arbeitgeber allein zu finanzierenden Anteil betrachtet, noch 1:5.

In Tab. 6 sind Kosten für eine Kalkulation auf der Ebene des Gesundheitssystems aufgeführt.

Danach liegen die Kosten für die 323 durchgeführten Koloskopien und 61 Polypektomien bei ca. 50000,- EUR Auf der anderen Seite wurden ca. 700000,- EUR an Therapie- und Krankenhauskosten durch die Vorsorgeaktion eingespart. Damit liegt die Kosten-Nutzen-Relation mit einem Verhältnis von 1:14 sogar höher als bei der betriebswirtschaftlichen Kalkulation.

\section{Diskussion}

Obwohl es in Deutschland seit mehr als 25 Jahren ein Programm zur Früherkennung gibt, nutzt im Durchschnitt nur jede dritte Frau (33\%) und nur jeder fünfte bis sechste Mann über 45 Jahre (18-20\%) die jährliche Krebsvorsorgeuntersuchung $(3,5,11)$.

In einer Umfrage des Allensbach-Institutes für Demoskopie aus dem Jahr 2002 (6) werden als Gründe für die schlechte Akzeptanz von Krebsvorsorgeuntersuchungen bei Männern Ängstlichkeit und
Tab.6 Kosten für Diagnostik, Therapie und Nachsorge kolorektaler Karzinome und deren Vorstufen (Quelle: FORTISNOVA Betriebskrankenkasse, Ludwigshafen).

\begin{tabular}{|c|c|}
\hline Leistung & Kosten \\
\hline Hohe Koloskopie & ca.150,- EUR \\
\hline Polypektomie (PE) & ca. 20,-EUR \\
\hline $\begin{array}{l}\text { Operation nach DRG, GO2B (Kolon-Teilresektion, } \\
\text { niedriger Schweregrad, } 14 \text { Tage Aufenthalt in der Klinik) }\end{array}$ & 6500,- EUR \\
\hline Rehabilitation ca. 21 Tage à 90,- EUR & 1900,- EUR \\
\hline $\begin{array}{l}\text { Nachsorge (Ärztliche Vorstellung, Untersuchungen, } \\
\text { Chemotherapie, Bestrahlung) }\end{array}$ & ca. 3000 ,- EUR \\
\hline DRG: Diagnosis Related Groups & \\
\hline
\end{tabular}

„Nicht-wissen-wollen“ genannt. Es fehle „die ausreichende Information über den Ablauf der Untersuchungen sowie deren Ziele“. Viele Männer bezweifeln den echten Nutzen der Vorsorge und sind der Meinung, dass sich Krebserkrankungen - einmal ausgebrochen nicht mehr erfolgreich behandeln lassen (6). Der Besuch beim Arzt erfolgt erst dann, wenn bereits Krankheitssymptome aufgetreten sind.

Die Haus- und Fachärzte sollten motiviert werden, ihren Patienten Grund und Ablauf der anstehenden Untersuchung und die Möglichkeit der medikamentösen Anxiolyse und Analgesie vor bzw. während des endoskopischen Eingriffs zu erläutern. Hier sind die Kassenärztlichen Vereinigungen und Dachverbände gefragt, z.B. ein Abrechnungssystem zu schaffen, das Präventivmedizin attraktiv macht und das ausführliche Beratungsgespräch im Arzt-Patienten-Kontakt aufwertet.

Um die Bevölkerung für die Präventionsmedizin dauerhaft zu sensibilisieren, bedarf es einer erhöhten Präsenz seriöser medizinischer Beiträge und Werbeaktionen in den Medien. Zum Thema „Darmkrebs“ gibt es qualitativ hochwertiges Informationsmaterial der Stiftung LebensBlicke, der Felix-Burda-Stiftung und aktuell eine Broschüre des Zweiten Deutschen Fernsehens mit Unterstützung der Stiftung LebensBlicke, des Deutschen Hausärzteverbandes und mehrerer deutscher Großunternehmen, in denen Indikation, Untersuchungsablauf und der Nutzen einer endoskopischen Untersuchung ausführlich geschildert werden.

Das bayerische Modellprojekt zur Darmkrebsfrüherkennung im Jahr 1996 hatte bereits gezeigt, dass eine Erhöhung der Beteiligung an Krebsvorsorgeuntersuchungen in der Bevölkerung generell möglich ist. Niedergelassene Haus- und Fachärzte wurden durch die Einführung einer Sondervergütung für die Durchführung des FOBT bei ihren Patienten motiviert. Zusätzlich wurde die Aktion vor allem in regionalen Medien intensiv beworben. Die Beteiligung an den Vorsorgeuntersuchungen stieg daraufhin bei Frauen von 28\% auf $47 \%$ und bei Männern von $12 \%$ auf 23\%, sank aber nach Beendigung der Aktion auf den gleichen Stand wie zuvor $(3,5,14)$.

Analog dazu gelang es in der BASF-Darmkrebs-Vorsorgeaktion ebenfalls nur mit großem persönlichen Einsatz und Einbindung aller firmeneigenen Medien eine Teilnahmequote von mehr als $30 \%$ der Zielgruppe zu erreichen.

Die Neuerkrankungsrate bei Darmkrebs kann aber nicht nur durch die Erhöhung der Beteiligung an Vorsorgeuntersuchungen nachhaltig gesenkt werden. Die sich aus pathologischen Untersuchungsergebnissen ergebende Folgediagnostik muss vom Arzt und den Pati- 
enten konsequent nach den Grundsätzen der evidenzbasierten Medizin durchgeführt werden. Bei positivem FOBT müssen immer Darmpolypen als Blutungsquelle in einer hohen Koloskopie ausgeschlossen werden, da diese Untersuchung mit 95\% die höchste Sensitivität und mit 100\% die höchste Spezifität für die Erkennung eines kolorektalen Karzinoms bzw. eines Adenoms besitzt. (4,9,11,12). Die jährliche Durchführung des FOBT wird ab einem Alter von 50 Jahren empfohlen (12). In unserer Vorsorgeaktion wurden knapp $20 \%$ der adenomatösen Polypen bereits in der Altersgruppe der 45bis 49- Jährigen entdeckt. Nur bei endoskopisch unauffälligem Dickdarmbefund können z.B. das Hämorrhoidalleiden, eine Antikoagulationstherapie mit Marcumar oder die Zufuhr eisenhaltiger Nahrungsmittel als verursachendes Agens für den positiven Ausfall des Tests angenommen werden.

Die alleinige Kontrolle des FOBT bis zum negativen Ergebnis trägt der Sensitivität des Testes mit ca. 40\% $(3,4,7,11)$ nicht ausreichend Rechnung. Darmpolypen bluten intermittierend und nicht zwangsläufig kontinuierlich. Die Spezifität des FOBT von mehr als $90 \%(3,4,11)$ stellt die eindeutige Indikation für eine sich anschließende endoskopische Diagnostik.

In unserer Untersuchung zeigte sich, dass das weitere Vorgehen von Betroffenen und/oder weiterbehandelnden Hausärzten von der Befundkonstellation abhängig war: Die endoskopische Abklärung erfolgte bei einem positiven FOBT häufiger als beim alleinigen Vorliegen einer positiven Familienanamnese. Lagen beide Befunde in Kombination vor, war die endoskopische Abklärungsrate mit 64,8\% am höchsten. Das alleinige Vorliegen einer familiären Belastung mit Darmkrebserkrankungen hatte für den behandelnden Arzt und den Betroffenen offenbar nicht genug „Druck“ erzeugt, um die aufwändige und vermeintlich unangenehme Koloskopie durchzuführen zu lassen. Jeder Teilnehmer der BASF-Vorsorgeaktion mit einer Indikation zur Koloskopie wurde seitens der Abteilung Arbeitsmedizin und Gesundheitsschutz mehrfach beraten und über das möglicherweise erhöhte Risiko des Vorliegens einer bösartigen Darmerkrankung aufgeklärt. Dennoch ließen weniger als 50\% der Teilnehmer die Untersuchung durchführen.

Die Anzahl der endoskopisch diagnostizierten Krebsvorstufen und die der manifesten Krebserkrankungen ist erschreckend hoch, zeigt aber letztendlich den Erfolg dieser Aktion. Unter der Annahme, dass bei den Nichtteilnehmern der Zielgruppe und denen, die trotz Indikation zur Koloskopie die Untersuchung nicht haben durchführen lassen, eine ähnlich hohe Zahl pathologischer Dickdarmbefunde besteht, lässt sich das Potenzial der sekundären Präventionsmedizin allein im Bereich der kolorektalen Karzinome ermessen. Die firmeninterne Kosten-Nutzen-Relation beträgt nur durch die Vermeidung von Arbeitsausfalltagen ein Vielfaches der angefallenen Kosten der Aktion. Ein noch höherer Nutzen ergibt sich bei der Kalkulation auf der Ebene des öffentlichen Gesundheitssystems. Derartige KostenNutzen-Berechnungen sind in der traditionellen Medizin nicht gern gesehen, da sie persönliche Schicksale der Patienten und den Behandlungsauftrag der Ärzte vermeintlich in den Hintergrund drängen. Andererseits aber können sie in Zeiten knapper Kassen und nicht enden wollender Diskussionen um die Reform des Gesundheitswesens ein Wegbereiter für die Intensivierung primärer und sekundärer Präventionsbemühungen sein. Krankheiten von vorneherein zu verhindern oder in ihren Frühstadien zu erkennen stellt immer noch die wirkungsvollste Variante einer Gesundheitsreform dar.
Fazit

Die Darmkrebsfrüherkennung verhindert schwerwiegende Erkrankungsfälle, die den Betroffenen und seine Angehörigen sowie auch den Arbeitgeber und das öffentliche Gesundheitswesen stark belasten. Die Mortalität an Darmkrebs kann nur gesenkt werden, wenn die Teilnahmequote an den entsprechenden Krebsvorsorgeuntersuchungen gesteigert wird und die Durchführung der endoskopischen Diagnostik bei Indikationsstellung konsequent erfolgt. In der betrieblichen Gesundheitsvorsorge sollten die Betriebs- und Werksärzte daher ihren engen Kontakt zum Arbeitnehmer im Sinne ihres Präventionsauftrages nutzen und entsprechende Zusatzuntersuchungen in Ergänzung zur gesetzlich vorgeschriebenen arbeitsmedizinischen Vorsorge anbieten. Die Mitarbeiter sehen in Vorsorgeaktionen eine positive Leistung des Unternehmens.

Unsere Erfahrungen zeigen, dass Aktionen wie diese nur mit hohem Aufwand an Organisation und Logistik in Zusammenarbeit mit den Haus- und Fachärzten in der Region erfolgreich durchgeführt werden können. Das Unternehmen unterstreicht damit die Fürsorgepflicht des Arbeitgebers und zeigt, dass ihm die Gesundheit der Mitarbeiter ein wichtiges Anliegen ist.

Autorenerklärung: Die Autoren erklären, dass sie keine finanziellen Verbindungen mit einer Firma haben, deren Produkt in dem Beitrag eine wichtige Rolle spielt (oder mit einer Firma, die ein Konkurrenzprodukt vertreibt).

Danksagung: Wir bedanken uns bei Herrn Oberarzt Dr. med. Dieter Schilling, Medizinische Klinik C, Klinikum der Stadt Ludwigshafen am Rhein gGmbH für wertvolle fachliche Ratschläge und begleitende Diskussionen.

Literatur

1 Arbeitsgemeinschaft Bevölkerungsbezogener Krebsregister in Deutschland (Hrsg.). Krebs in Deutschland, 3. Ausgabe, Saarbrücken, 2002

2 Brenner H, Arndt V, Stürmer T, Stegmaier C, Ziegler H, Dhom G. Präventionspotential endoskopischer Vorsorgeuntersuchungen für kolorektale Karzinome. Dtsch Ärztebl 2002; 33: A2186-A2192

3 Eickhoff A, Maar C, Birkner B, Riemann J. Dickdarmkrebs in Deutschland, Vorsorge und Früherkennungsmaßnahmen für Laien und Ärzte. Internist 2003; 44: 278und Frith

4 Eickhoff A, Reinacher-Schick A, Schmiegel W, Riemann J. Primärprävention, Screening und präventive Chirurgie, Kolorektalkarzinom. Dtsch Med Wochenschr 2002; 127: 543-545

5 Eickhoff A, Riemann J. Das Kolonkarzinom-Aktueller Stand der Früherkennung und endoskopischer Prävention. Internist 2000; 41: 860-867

6 Institut für Demoskopie Allensbach. Repräsentativerhebung- Men's Health-Pfitzer-Gesundheitsmonitor 2002 http://www.der-gesunde-mann.de/x/pdf/ gesundheitsmonitor_2002.pdf Stand: Sept. 2002,

7 Knöpnadel J, Altenhofen L, Brenner G. Epidemiologie und gesundheitsökonomische Bedeutung des Darmkrebses in Deutschland. Internist 2003; 44: 268-277

8 Kullmann F. Karzinogenese und heriditäre Kolonkarzinome. Internist 2003; 44: 254-267

9 Liebermann DA, Weiss DG, Bond JH et al. Use of colonoscopy to screen asymptomatic adults for colorectal cancer. N Engl J Med 2000; 343: 162-168

10 Mandel JS, Church TR, Bond JH, Ederer F, Geisser MS. The effect of fecal-occultblood screening on the incidence of colorectal cancer. N Engl J Med 2000; 343: 1603-1607

11 Pox C, Schulmann K, Schmiegel W. Konventionelles und molekulares Screening (Stuhltests). Internist 2002; 44: 287-293

12 Schmiegel W, Adler G, Frühmorgen P et al. Kolorektales Karzinom: Prävention und Früherkennung in der asymptomatischen Bevölkerung - Vorsorge bei Risikopatienten - Endoskopische Diagnostik, Therapie und Nachsorge von Polypen und Karzinomen. Z Gastroenterol 2000; 38: 49-75

13 Schön D, Bertz J, Görsch B et al. Gesundheitsberichterstattung für Deutschland, Schwerpunktbericht, Entwicklung und Überlebensraten von Krebspatienten in Deutschland Robert Koch-Institut, 1999

14 Zentralinstitut für die kassenärztliche Versorgung in der Bundesrepublik Deutschland. Modellprojekt Früherkennung des kolorektalen Karzinoms 1999 